\title{
Wavelength-encoded tomography based on optical temporal Fourier transform
}

\author{
Chi Zhang and Kenneth K. Y. Wong ${ }^{\text {a) }}$ \\ Photonic Systems Research Laboratory, Department of Electrical and Electronic Engineering, \\ The University of Hong Kong, Pokfulam Road, Hong Kong
}

(Received 24 June 2014; accepted 13 August 2014; published online 4 September 2014)

\begin{abstract}
We propose and demonstrate a technique called wavelength-encoded tomography (WET) for noninvasive optical cross-sectional imaging, particularly beneficial in biological system. The WET utilizes time-lens to perform the optical Fourier transform, and the time-to-wavelength conversion generates a wavelength-encoded image of optical scattering from internal microstructures, analogous to the interferometery-based imaging such as optical coherence tomography. Optical Fourier transform, in principle, comes with twice as good axial resolution over the electrical Fourier transform, and will greatly simplify the digital signal processing after the data acquisition. As a proofof-principle demonstration, a $150-\mu \mathrm{m}$ (ideally $36 \mu \mathrm{m}$ ) resolution is achieved based on a $7.5-\mathrm{nm}$ bandwidth swept-pump, using a conventional optical spectrum analyzer. This approach can potentially achieve up to $100-\mathrm{MHz}$ or even higher frame rate with some proven ultrafast spectrum analyzer. We believe that this technique is innovative towards the next-generation ultrafast optical tomographic imaging application. (c) 2014 AIP Publishing LLC.
\end{abstract}

[http://dx.doi.org/10.1063/1.4893948]

Tomographic imaging technologies such as the computed tomography by $\mathrm{x}$-ray, ${ }^{1}$ magnetic resonance imaging (MRI), ${ }^{2}$ ultrasound imaging, ${ }^{3}$ and optical coherence tomography $(\mathrm{OCT})^{4}$ have found widespread applications in microscale bio-medical imaging. Among these technologies, optical tomography system (e.g., OCT) is advantageous in terms of resolution, owing to its short wavelength. ${ }^{4,5}$ Optical frequency is far beyond the detectable range of electrical means, and OCT system leverages the low frequency beating introduced by the interferometer to differentiate the reflective depth. ${ }^{4}$ On the other hand, some straightforward methods like the radio detection and ranging (RADAR) or the optical time domain reflectometer (OTDR) ${ }^{6}$ can only measure large-scale distance, where the resolution is limited by the bandwidth of the oscilloscope. Fortunately, an ultrafast optical oscilloscope is available with the advance of the time-lens approach, which resolved 200-fs short pulse in the spectral domain. ${ }^{7}$ The combination of these two schemes (OTDR and the timelens oscilloscope) can directly convert the tiny depth difference into wavelength shift, which provides a promising solution for the advanced ultrafast tomographic applications, and we call it wavelength-encoded tomography (WET). This technique overcomes the inherited speed limitation of the OCT, and also potentially improves the resolution in performing non-invasive cross-sectional imaging.

However, the femtosecond pulse required by the timelens oscilloscope in Ref. 7 will inevitably encounter undesirable nonlinear effects in biological samples, which will affect the imaging quality of the linear scattering system (e.g., OCT). Therefore, the single time-lens focusing structure in Ref. 7 is adjusted to two time-lenses, with opposite focal group-dispersion delays (GDDs), to avoid the ultra-short

\footnotetext{
${ }^{\text {a) }}$ Author to whom correspondence should be addressed. Electronic mail: kywong@eee.hku.hk
}

pulse interacting with the sample. Figure 1 shows the temporal ray diagram of the WET configuration, which can be understood by the space-time duality. ${ }^{8}$ Here, the spatial distance $(d)$ along the vertical axis corresponds to the temporal delay $(\Delta t)$, while the spatial axial angle $(\theta)$ corresponds to the temporal wavelength $(\Delta \lambda)$. In Fig. 1(a), one converging lens and one diverging lens with identical focal length $(f)$ are aligned with each other, and tilting of the axial angle $(\theta=d / f)$ is achieved by the offset $(d)$ between these two lenses along the vertical axis (Fig. 1(b)). ${ }^{9}$ Therefore, in the temporal domain, these two corresponding time-lenses can realize the linear optical mapping between the reflective temporal delay $(\Delta t=2 d / c)$ and the wavelength $(\Delta \lambda)$. More importantly, no short pulse is involved throughout this process, and the energy is distributed uniformly along the time span.

From the practical point of view, these two time-lenses are implemented with the swept-pumped four-wave mixing (FWM) process (Figs. 1(c) and 1(d)), owing to the required large swept bandwidth and less than gigahertz frame rate. ${ }^{10,11}$ Here, the identical focal GDD $\left(\Phi_{f}\right)$ are achieved with the same swept-pump, while the converging and diverging features are realized with different parametric mixing directions, from short wavelength to long wavelength and vice versa. After time-lens 1 , the newly generated idler is launched to illuminate a reflective sample, which will scatter the idler along the temporal axis before combining together with the same swept-pump in time-lens 2. Ideally, the timelens 2 will generate a quasi-CW source as the input signal, and the delayed idler will change its wavelength, as the dashed and dotted lines shown in Fig. 1(d). This mechanism provides a linear relation between the reflection depth $(d)$ and the output wavelength shift $(\Delta \lambda)$ as follows:

$$
\Delta \lambda=\frac{\lambda_{0}^{2}}{2 \pi c} \cdot \frac{\Delta t}{\Phi_{f}}=\frac{4}{c D L} d=\varepsilon \cdot d,
$$


(a)

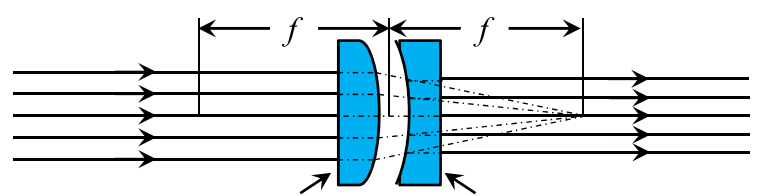

(Converging) Lens 1

(b)

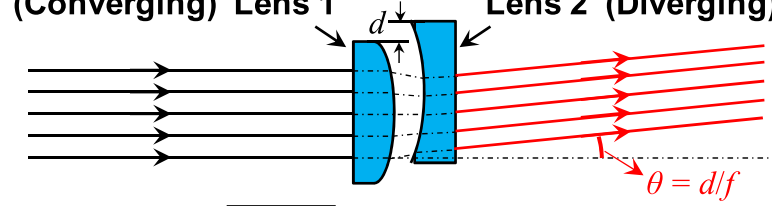

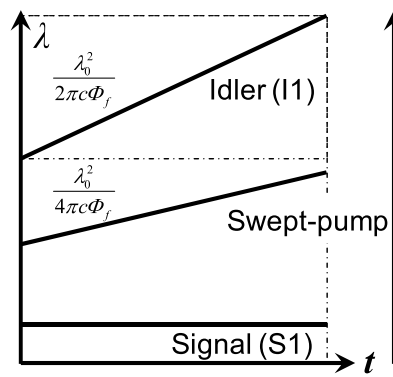

(d) (c) Time-lens 1

\section{Time-lens 2}

$$
\Delta \lambda=\frac{\lambda_{0}^{2}}{2 \pi c \Phi_{f}} \cdot \frac{2 d}{c}
$$

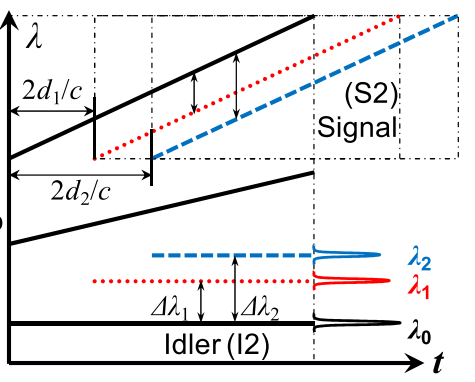

FIG. 1. Principle of the depth-to-wavelength conversion by WET system. Based on the space-time duality, the spatial axial angle corresponds to the wavelength in the temporal domain. (a) and (b) In the spatial domain, tilting the axial angle of a parallel beam by the offset between two complementary lenses (one converging lens and one diverging lens with identical focal length) along the vertical axis. (c) and (d) In the temporal domain, two complementary time-lenses are implemented by the swept-pumped FWM process, and the slope of the swept-pump and the idler (I1) are shown in Fig. 1(c). The temporal offset delay is introduced by the depth reflective sample, thus different spatial depth in the reflective sample will convert to different wavelength at the output.

where $c$ is light velocity, $D L$ is the swept-pump dispersion (suppose it is generated from stretched pulse), and $\varepsilon=4 / c D L$ is the depth-to-wavelength ratio. The detailed derivation of $\varepsilon$ can be obtained from Fig. 1(d) and Ref. 11.

Figure 2 illustrates the detailed experimental setup of these two time-lenses. A pulsed source (500-fs pulsewidth, $\lambda_{B W}=7.5 \mathrm{~nm}$ ) passes through a spool of $1-\mathrm{km}$ single-mode fiber (SMF-28, $D L=17 \mathrm{ps} / \mathrm{nm}$ ) to generate the swept-pump for the two-stage FWMs. ${ }^{10,11}$ Here, the FWM leverages the third-order nonlinear susceptibility in two spools of $50-\mathrm{m}$ highly-nonlinear dispersion-shifted fibers (HNL-DSFs), with the zero-dispersion wavelength at $1554 \mathrm{~nm}$. In the first-stage FWM (the left shaded box in Fig. 2), 1-mW CW signal (S1) and the swept-pump (amplified to $100 \mathrm{~mW}$ ) generate the idler (I1) with doubled swept range, as shown in Fig. 3(a). This idler (I1, $0.5 \mathrm{~mW}$ ) is filtered out by a wavelengthdivision multiplexing (WDM) filter, and amplified to $5 \mathrm{~mW}$ by an L-band Erbium doped fiber amplifier (EDFA) before illuminating the sample.

The sample arm introduces $\sim 10$-dB loss, and different reflection depths scatter the idler (I1) with corresponding temporal delays. Here an optical delay line in the sample arm synchronizes the scattered idlers ( 2 2, as the signal for the second-stage FWM) with the second swept-pump. Therefore, after the second-stage FWM, the idler (I2, 0.2

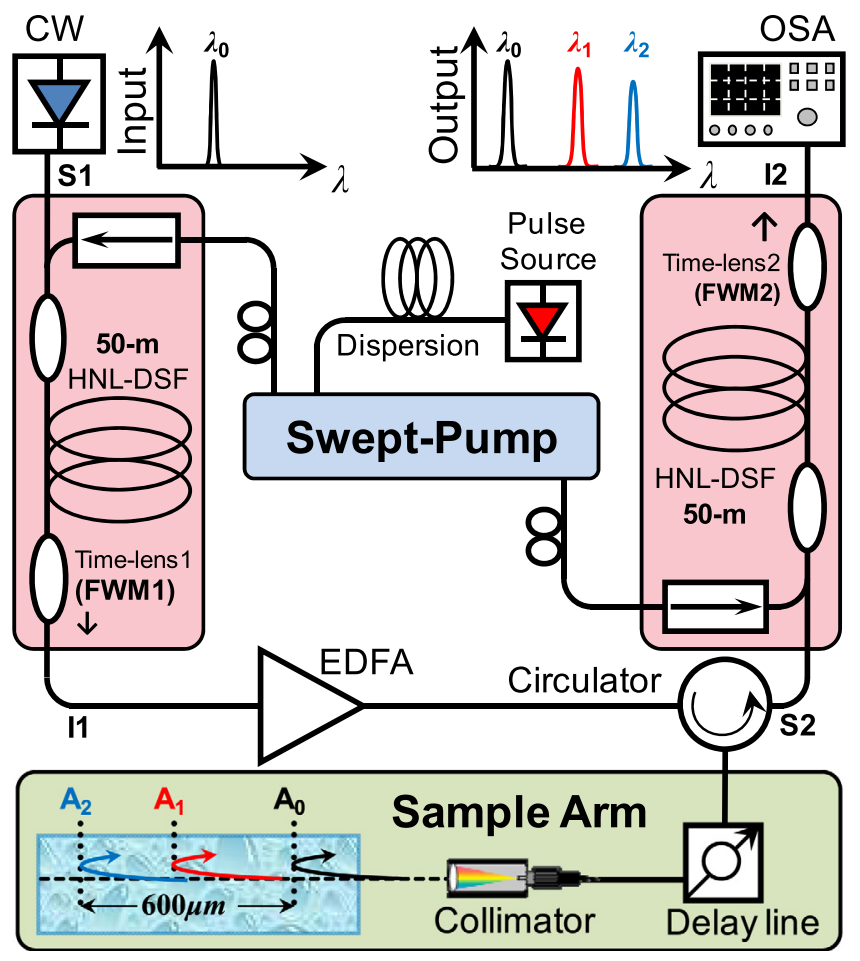

FIG. 2. Experimental setup of the WET system. The two-stage FWMs are based on two spools of $50-\mathrm{m}$ HNL-DSF, and they share the identical sweptpump, which is generated from the dispersive stretched pulse source. The idler (I1) of the first-stage FWM comes to the sample arm, and the reflective delayed idler (S2) is coupled into the second-stage FWM as the signal. The newly generated idler (I2) is the output signal and is detected by the OSA, since the depth information is encoded on the wavelength.

$\mathrm{mW}$ ) is generated at the original signal wavelength, as shown in Fig. 3(b). The idler (I2) spectrum, captured by a conventional optical spectrum analyzer (OSA, Yokogawa AQ6370C, 0.05-nm resolution), can manifest the spatial information ( $A_{0}, A_{1}$, and $A_{2}$ in the sample arm of Fig. 2), and shown as different colors $\left(\lambda_{0}, \lambda_{1}\right.$, and $\left.\lambda_{2}\right)$ in the output spectra of Fig. 2. Moreover, the two time-lenses configuration employs a CW source as the signal, and no synchronization
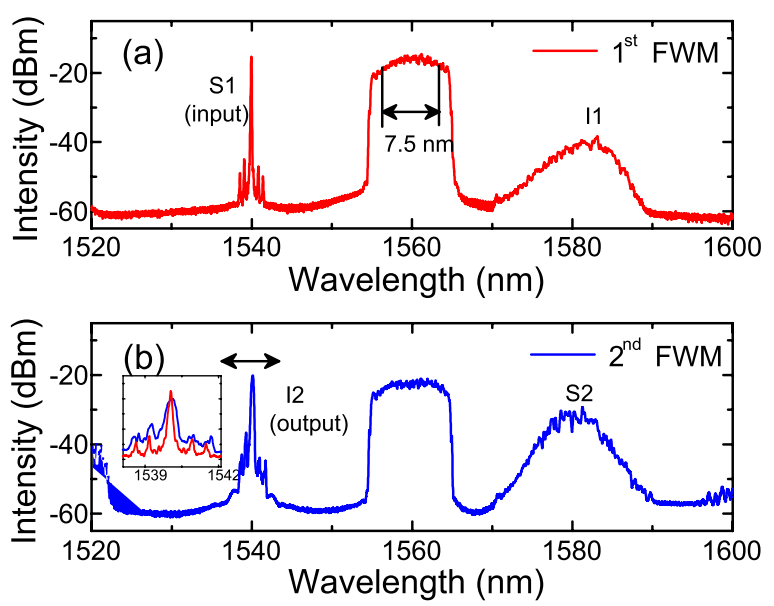

FIG. 3. Spectra of the two-stage FWM in the WET system. (a) A CW signal (S1) and a swept-pump generate an idler (I1) with doubled swept range. (b) Temporally delayed and re-amplified idler (S2) is combined with the identical swept-pump to retrieve a $\mathrm{CW}$ idler (I2) overlap with the input $\mathrm{CW}$ source. The inset shows the spectrum broadening of the retrieved $\mathrm{CW}$ idler over the original CW signal. 


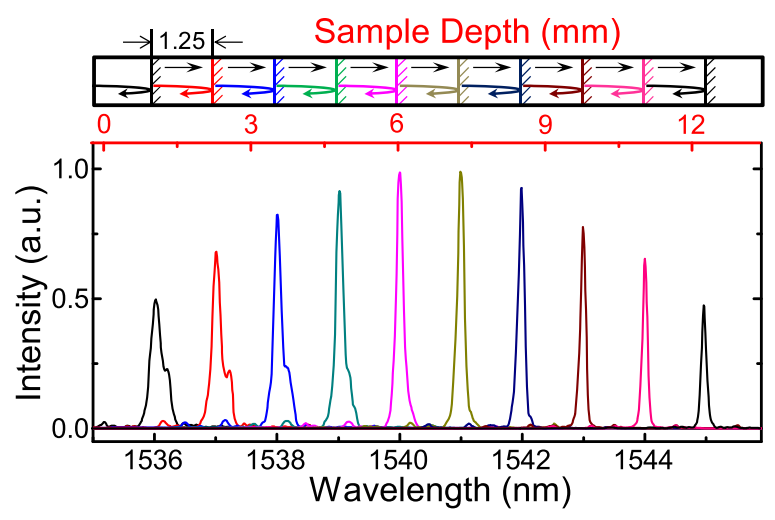

FIG. 4. Characterization of the depth-to-wavelength interpretation in WET system. By changing the spatial depth of a reflective mirror in the sample arm, with around 1-mm separation and across 11-mm range, the wavelength of the retrieved CW ilder (I2) also varies from $1536 \mathrm{~nm}$ to $1545 \mathrm{~nm}$, which matches well with their linear mapping relation. These traces are captured by a conventional OSA, with $5-\mathrm{Hz}$ frame rate.

is required between the signal and pulsed pump, compared with Ref. 7. The overall setup can be pictorially viewed as a "lock" system: the FWM1 locks a swept-pump onto the CW signal, and the FWM2 using the same swept-pump as a "key" to unlock the temporal delay (i.e., reflection depth information) from the wavelength shifting.

Then, we evaluated the layers resolving performance of the WET system. According to the relation shown in Eq. (1), the output wavelength shift $(\Delta \lambda)$ is directly proportional to the reflection depth $(d)$. To quantitatively characterize this feature, a precision translation stage was introduced to control the reflective mirror, which was moved with $1.25-\mathrm{mm}$ separation across $11-\mathrm{mm}$ range, and the results are shown in Fig. 4. It can be observed that the 3-dB observation range was up to $1 \mathrm{~cm}$ and the depth-to-wavelength mapping ratio was $\varepsilon=0.8 \mathrm{~nm} / \mathrm{mm}$, matched well with Eq. (1). In this WET system, the spatial resolution $\left(R_{W E T}\right)$ refers to the capability of an optical imaging system to distinguish different layers, which can be calculated with the spectral width $(\delta \lambda)$ and the mapping ratio $(\varepsilon)$. The ideal $\delta \lambda$ is determined by the temporal pulsewidth $\left(\lambda_{B W} D L\right)$, which will be narrowed by the FWM process. ${ }^{10}$ Noted that $\delta \lambda$ is also limited by the high frequency intensity fluctuation of the swept-pump. Suppose there is no intensity fluctuation over the envelope of the swept-pump, and phase-matching condition is satisfied over the whole conversion range, then the spatial resolution of the WET system can be shown as

$$
R_{W E T}=\frac{\delta \lambda}{\varepsilon}=\frac{\lambda_{0}^{2}}{2 \pi c} \cdot \frac{4 \ln 2}{\lambda_{B W} D L} \cdot \frac{c D L}{4}=\frac{\ln 2 \lambda_{0}^{2}}{2 \pi \lambda_{B W}},
$$

where $\lambda_{B W}$ is the full-width at half-maximum (FWHM) of the swept-pump spectrum. Based on the experimental pump bandwidth $\left(\lambda_{B W}=7.5 \mathrm{~nm}\right)$, the ideal spatial resolution should be $36 \mu \mathrm{m}$. Compared with OCT, ${ }^{4}$ the WET system can, in principle, achieve four times sharper resolution with the same bandwidth $\left(\lambda_{B W}\right)$ based on Eq. (2). The intuitive understanding can be briefly explained as follows: First, the doubled frequency relation from the swept-pump to the idler in the FWM (i.e., the idler bandwidth can be doubled of the pump), which contributes a factor of two; second, the optical
Fourier transform (i.e., WET) achieves better temporal resolution over the digital (electrical) Fourier transform (i.e., OCT) by another factor of two. Specifically, the optical Fourier transform by the time-lens has taken into account of the phase term, while the electrical Fourier transform in OCT is performed after the square-law photodetector, which only considers the intensity part. The second factor of two is guaranteed, while the first factor (conversion bandwidth) might be limited by the phase-matching condition of the FWM. ${ }^{12}$

The spectra of these two-stage FWMs are shown in Fig. 3, and it is noted that the idler (I1) bandwidth $(10 \mathrm{~nm})$ is not ideally doubled as that of the swept-pump $(7.5 \mathrm{~nm})$, owing to the higher-order dispersion induced phase mismatch. Therefore, the conversion bandwidth also limited the resolution, and the phase matching condition requires shorter HNL-DSF with lower dispersion coefficient $(D)$ and slope $(S)$. Some advanced dispersion-engineered nonlinear medium, such as the silicon waveguide, helps to achieve 100nm FWM wavelength conversion bandwidth, ${ }^{13}$ which will benefit the implementation of the WET system in the near future. The inset of Fig. 3(b) shows the comparison between the input and the output spectra, which is broadened from $0.05 \mathrm{~nm}$ (OSA resolution) to $\delta \lambda=0.12 \mathrm{~nm}$. Two factors contribute to this spectral broadening effect: first, the phasemismatching decreases the pulsewidth of idler (I1) by $30 \%$, since its bandwidth is degraded from $15 \mathrm{~nm}$ to $10 \mathrm{~nm}$ after FWM1 (as shown in Fig. 3(a)). Considering the same narrowing effect in FWM2, the pulsewidth of idler (I2) will decrease by half, namely broaden the spectral width by a factor of two (from $0.03 \mathrm{~nm}$ to $0.06 \mathrm{~nm}$ ). Second, the temporal intensity fluctuation of the swept-pump introduced another factor of two to the spectral width of the idler (I2) from $0.06 \mathrm{~nm}$ to $0.12 \mathrm{~nm}$. According to the measurement in Fig. 4 (based on the OSA with 5-Hz frame rate), the corresponding resolution of our WET system is $R_{W E T}=\delta \lambda / \varepsilon=150 \mu \mathrm{m}$, which is four times degraded from the ideal case in Eq. (2).

To capture the 2D cross-sectional image, a galvo mirror (M1) is proposed to scan in the sample arm as shown in the top box of Fig. 5. The two time-lenses WET system can optically convert the depth information to spectrum, and another essential part is capturing the spectrum to construct the $2 \mathrm{D}$ image. In this sense, it is similar to the spectral-domain OCT (SD-OCT), which also requires a spectrometer to capture the spectral information. ${ }^{14,15}$ However, the spectrum in SD-OCT carries the interference fringes, and further Fourier transform is required to retrieve the depth information. While in the WET system, the spectrum directly generates the depth information (with better resolution over the SD-OCT), as long as it can be captured by some synchronized single-shot spectrometer, ${ }^{16,17}$ this WET technique can be configured as an ultrafast tomographic system. To fully utilize the $100-\mathrm{MHz}$ frame rate of the WET, here we introduce two methods: First, dispersing the spectrum onto a spatial CCD sensor (Fig. 5(a)) and also combining with another scanning galvo mirror (M2) along the horizontal direction to construct the 2D image. M1 and M2 are synchronized by the identical driving signal, and the B-scan rate is only determined by the frame rate of the CCD sensor (as high as $\sim \mathrm{kHz}$ ). ${ }^{18}$ Second, we can utilize a recently demonstrated ultrafast optical spectrum analyzer, 


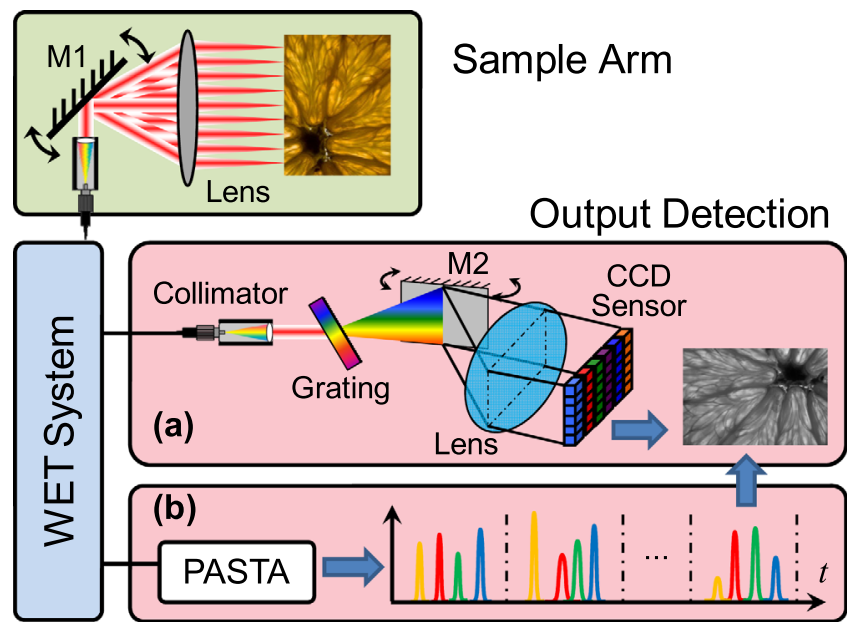

FIG. 5. Proposed data acquisition schemes for the WET system. The depth information is encoded to the output wavelength, and a spectrometer is required to capture the data. In the sample arm, a galvo mirror M1 scans the sample along the cross-sectional direction. There are two high-speed acquisition methods: (a) The spectral information is spatially dispersed by a diffractive grating, and a galvo mirror M2 is synchronized with the sample arm mirror M1. The 2D cross-sectional image can then be directly captured by a CCD sensor. (b) A synchronized PASTA system is introduced here, which directly converts the wavelength information to the temporal domain. These frames can be reconstructed to the 2D cross-sectional image (an image of orange vesicles is employed here for illustration purpose).

parametric spectro-temporal analyzer (PASTA), with 0.02nm resolution. ${ }^{17,19}$ Actually, the PASTA system can share the same pump source of the WET system, which naturally synchronizes these two systems together, therefore fully utilizes the 100-MHz A-scan rate, as shown in Fig. 5(b).

With these proposed ultrafast spectral acquisition modalities, we can approximately compare the OCT and WET systems as shown in Table I. They are compared under the same spectral bandwidth $(7.5 \mathrm{~nm})$, and the WET system achieves the same resolution as the OCT, even with 4 times degradation. There are two directions in narrowing the spectral width: extending the conversion bandwidth, and reducing the intensity fluctuation of the swept-pump. Therefore, our future work will include developing a more stable swept-

TABLE I. Comparison between the OCT and WET system under the same spectral width $(7.5 \mathrm{~nm})$.

\begin{tabular}{lcc}
\hline \hline & OCT & WET \\
\hline Spectral width $\left(\lambda_{B W}\right)$ & $7.5 \mathrm{~nm}^{\mathrm{a}}$ & $7.5 \mathrm{~nm}^{\mathrm{b}}$ \\
Resolution $(R)$ & $R_{O C T}=\frac{2 \ln 2 \lambda_{0}^{2}}{\pi \lambda_{B W}}$ & $R_{W E T}=\frac{\ln 2 \lambda_{0}^{2}}{2 \pi \lambda_{B W}}$ \\
Theoretic $R$ & $144 \mu \mathrm{m}$ & $36 \mu \mathrm{m}$ \\
Measured $R$ & $150 \mu \mathrm{m}^{\mathrm{c}}$ & $150 \mu \mathrm{m}$ \\
Depth range & $>1 \mathrm{~cm}^{\mathrm{m}}$ & $>1 \mathrm{~cm}$ \\
A-scan rate $\left(f_{A}\right)$ & $40 \mathrm{kHz}^{\mathrm{d}}$ & $100 \mathrm{MHz}$ \\
\hline \hline
\end{tabular}

${ }^{\mathrm{a}}$ Over $100 \mathrm{~nm}$ is available, and it can be incoherent source in the SD-OCT, or Fourier domain mode-locking (FDML) laser source ${ }^{20}$ and some semiconductor swept-source with micro-electromechanical mirror systems (MEMS) based filter in swept-source OCT.

${ }^{\mathrm{b}} \mathrm{Up}$ to $10 \mathrm{~nm}$, which is limited by the phase-matching condition of our FWM waveguide.

${ }^{c}$ This is an estimated value, owing to the small resolution degradation introduced by the OCT system.

${ }^{\mathrm{d}}$ Mainly limited by the graphics processing unit processing speed (to perform the calibration and Fourier transform) in the SD-OCT. ${ }^{14,15,20}$ pump source, exploring a better nonlinear medium in achieving wideband FWM conversion, and implementing the detection schemes proposed in Fig. 5.

In this letter, we propose and demonstrate an optical tomographic system, WET, based on optical temporal Fourier transform, with $150-\mu \mathrm{m}$ resolution across $1-\mathrm{cm}$ depth range. The advantages of WET versus OCT include: (1) no separated reference branch is required in generating the interference pattern (fringes); (2) since the reference branch is not required, the imaging speed will no longer be limited by the mechanical component (such as moving the reference arm in time-domain OCT to generate the fringes) $;^{4}$ (3) it is a predominantly ultrafast all-optical process, since the output scattered signal directly reflects the spatial information, and no post-processing is required compared with the SD-OCT; (4) the WET system can, in principle, achieve four times sharper resolution than the OCT system with the same spectral bandwidth. Therefore, this WET system outlines a convincing path in improving the A-scan rate and the imaging quality, and also largely simplifying the system requirement. As a proof-of-principle effort, though suboptimal in its resolution, it demonstrates the essence of our proposed technique. We believe that this time-lens Fourier transform based tomography concept will not only contribute to the development of the optical imaging community but also enlighten the research areas where more promising imaging modalities are involved.

This work was partially supported by grant from the Research Grants Council of the Hong Kong Special Administrative Region, China (Project Nos. HKU 7172/12 E and HKU 17208414E). The authors also acknowledge Sumitomo Electric Industries for providing the HNL-DSF and Alnair for providing the variable bandwidth tunable band-pass filter (VBTBPF).

${ }^{1}$ G. N. Hounsfield, "Computerized transverse axial scanning (tomography): Part I. Description of system,” Br. J. Radiol 46, 1016-1022 (1973).

${ }^{2}$ R. Damadian, M. Goldsmith, and L. Minkoff, "NMR in Cancer: XVI. FONAR image of the live human body," Physiol. Chem. Phys. 9, 97-100 (1977).

${ }^{3}$ J. J. Wild and J. M. Reid, "Application of echo-ranging techniques to the determination of structure of biological tissues," Science 115, 226-230 (1952).

${ }^{4}$ D. Huang, E. A. Swanson, C. P. Lin, J. S. Schuman, W. G. Stinson, W. Chang, M. R. Hee, T. Flotte, K. Gregory, C. A. Puliafito, and J. G. Fujimoto, "Optical coherence tomography," Science 254, 1178-1181 (1991).

${ }^{5}$ W. Wieser, B. R. Biedermann, T. Klein, C. M. Eigenwillig, and R. Huber, "Multi-Megahertz OCT: High quality 3D imaging at 20 million A-scans and 4.5 GVoxels per second," Opt. Express 18, 14685-14704 (2010).

${ }^{6}$ B. Chance, J. S. Leigh, H. Miyake, D. S. Smiths, S. Nioka, R. Greenfeld, M. Finander, K. Kaufmann, W. Levy, M. Young, P. Cohen, H. Yoshioka, and R. Boretsky, "Comparison of time-resolved and -unresolved measurements of deoxyhemoglobin in brain," Proc. Natl. Acad. Sci. USA 85, 4971-4975 (1988).

${ }^{7}$ M. A. Foster, R. Salem, D. F. Geraghty, A. C. Turner-Foster, M. Lipson, and A. L. Gaeta, "Silicon-chip-based ultrafast optical oscilloscope," Nature 456, 81-84 (2008).

${ }^{8}$ B. H. Kolner, "Space-time duality and the theory of temporal imaging," IEEE J. Quantum Electron. 30, 1951-1963 (1994).

${ }^{9}$ A. E. Conrady, Applied Optics and Optical Design, Part One (Courier Dover Publications, 2011).

${ }^{10}$ C. Zhang, K. K. Y. Cheung, P. C. Chui, K. K. Tsia, and K. K. Y. Wong, "Fiber optical parametric amplifier with high-speed swept pump," IEEE Photonics. Technol. Lett. 23, 1022-1024 (2011). 
${ }^{11}$ C. Zhang, P. C. Chui, and K. K. Y. Wong, "Comparison of state-of-art phase modulators and parametric mixers in time-lens applications under different repetition rates," Appl. Opt. 52, 8817-8826 (2013).

${ }^{12}$ M. E. Marhic, Fiber Optical Parametric Amplifiers, Oscillators and Related Devices (Cambridge University Press, Cambridge, 2007).

${ }^{13}$ A. C. Turner-Foster, M. A. Foster, R. Salem, A. L. Gaeta, and M. Lipson, "Frequency conversion over two-thirds of an octave in silicon nanowaveguides," Opt. Express 18, 1904-1908 (2010).

${ }^{14}$ S. Yun, G. Tearney, J. de Boer, N. Iftimia, and B. Bouma, "High-speed optical frequency-domain imaging," Opt. Express 11, 2953-2963 (2003).

${ }^{15}$ R. A. Leitgeb, W. Drexler, A. Unterhuber, B. Hermann, T. Bajraszewski, T. Le, A. Stingl, and A. F. Fercher, "Ultrahigh resolution Fourier domain optical coherence tomography," Opt. Express 12, 2156-2165 (2004).
${ }^{16}$ D. R. Solli, J. Chou, and B. Jalali, "Amplified wavelength-time transformation for real-time spectroscopy," Nat. Photonics 2, 48-51 (2008).

${ }^{17}$ C. Zhang, J. Xu, P. C. Chui, and K. K. Y. Wong, "Parametric spectrotemporal analyzer (PASTA) for real-time optical spectrum observation," Sci. Rep. 3, 2064 (2013).

${ }^{18}$ T. G. Etoh, C. V. Le, Y. Hashishin, N. Otsuka, K. Takehara, H. Ohtake, T. Hayashida, and H. Maruyama, "Evolution of Ultra-High-Speed CCD Imagers," Plasma Fusion Res. 2, S1021 (2007).

${ }^{19}$ C. Zhang, X. Wei, and K. K. Y. Wong, "Performance of parametric spectrotemporal analyzer (PASTA)," Opt. Express 21, 32111-32122 (2013).

${ }^{20}$ R. Huber, M. Wojtkowski, and J. G. Fujimoto, "Fourier domain mode locking (FDML): A new laser operating regime and applications for optical coherence tomography," Opt. Express 14, 3225-3237 (2006). 